\title{
Identification of and antimicrobial resistance in bacteria causing caprine mastitis in three states and a city in Central Mexico under manual and mechanical milking conditions
}

\begin{abstract}
Mastitis can be caused by infectious agents, bacterial mastitis is the most common, in Mexico, and data regarding mastitis in goats are scarce. The aim of this work was to identify the bacteria most frequently present in goats with mastitis and assess isolate susceptibility to chemotherapeutic agents. A total of 460 samples of goat milk were obtained from the states of Guanajuato, Querétaro, and Morelos and Mexico City. A total of 56 bacteriapositive samples were obtained. Of these bacteria-positive samples, 33 were identified as belonging to the Staphylococcus genus, representing $58.92 \%$ of the positive samples. Of the 33 Staphylococcus-positive samples, 16 were identified as S. epidermidis, representing $48.48 \%$ of the Staphylococcus-positive samples; 5 each were identified as S. simulans and S. xylosus, each representing $15.15 \%$ of the Staphylococcus-positive samples; 3 were identified as S. lentus, representing $9.09 \%$ of the Staphylococcus-positive samples; 2 were identified as S. aureus, representing $6.06 \%$ of the Staphylococcus-positive samples; and 1 each was identified as S. caprae and S. cohnii, each representing $3.03 \%$ of the Staphylococcus-positive samples. Resistance to streptomycin, ampicillin, polymyxin B, tetracycline and tobramycin was assessed using an antibiogram on all specimens that were Staphylococcus-positive. The differences observed in the efficacies of the evaluated drugs illustrate the importance of carrying out tests for the identification of and evaluation of antimicrobial susceptibility in bacterial agents related to mastitis
\end{abstract}

Keywords: goats, mastitis, coagulase-negative staphylococci, antibiogram
Volume 7 Issue $3-2018$

\author{
Stephany Patricia Manzanero Martínez,' \\ Rocio Angélica Ruiz Romero, ${ }^{2}$ Roberto \\ Arnulfo Cervantes Olivares, ${ }^{3}$ Valentín Efrén \\ Espinosa Ortiz ${ }^{4}$ Andrés Ernesto Ducoing \\ Watty ${ }^{2}$ \\ 'Center for Practical Education, Research, Production and \\ Animal Health, National Autonomous University of Mexico, \\ Mexico \\ ${ }^{2}$ Department of Medicine and Zootechnics of Ruminants, \\ National Autonomous University of Mexico, Mexico \\ ${ }^{3}$ Department of Microbiology and Immunology, National \\ Autonomous University of Mexico, Mexico \\ ${ }^{4}$ Department of Economics, National Autonomous University of \\ Mexico, Mexico City, Mexico
}

\begin{abstract}
Correspondence: Rocío Angélica Ruiz Romero, Department of Medicine and Zootechnics of Ruminants, Faculty of Veterinary Medicine and Zootechnics, National Autonomous University of Mexico, Av. Universidad 3000, Delegación Coyoacán, Col. Ciudad Universitaria, C.P. 04510 Mexico City, Mexico, Tel 52 (55) 5622 59 72, Email rarr2212@unam.mx
\end{abstract}

Received: February 22, 2018 | Published: June 12, 2018

\section{Introduction}

In Mexico, the caprine population has been estimated at 8.6 million goats. ${ }^{1}$ In recent years, there has been an increase in the production of goat milk and demand for its derivatives; ${ }^{2}$ therefore, it is necessary to have sufficient milk that is safe and of good nutritional quality. Mastitis is defined as inflammation of the mammary glands that can be caused by trauma or infectious agents, such as bacteria, fungi and viruses; of these aetiologies, bacterial mastitis is the most common. This disease affects the physical, chemical and bacteriological characteristics of milk. Clinical mastitis is characterized by pathological changes in the mammary gland, such as inflammation, loss of the ability to produce milk and alterations in the color and consistency of the milk. ${ }^{3}$ Clinical mastitis has been found to have a low incidence in goats, which may reach up to $2 \%$ per year. ${ }^{4}$ In the case of subclinical mastitis, there are no visible signs of disease since the milk exhibits a normal appearance. Accordingly, this disease may only be detected through the use of diagnostic techniques based on the quantification of somatic cells in the milk. Mastitis transmission can occur from goat to goat and via the hands of milkers, tea cups, beds and goat kids. ${ }^{5}$ Included among the most common bacteria that may cause caprine mastitis are Staphylococcus spp., Streptococcus spp., enterobacteria and Mycoplasma spp. ${ }^{6}$ The most commonly identified genus is Staphylococcus, within which the most important species are Staphylococcus aureus and coagulase-negative staphylococci (CoNS). $S$. aureus produces clinical mastitis and is responsible for gangrenous mastitis, which is caused by $\alpha$ toxin; in goats, infection with this agent can lead to mortality within 24 hours if a rapid and effective treatment is not initiated. S. aureus is considered a public health hazard due to the toxins that it produces and the reductions in milk production it causes. ${ }^{5}$ The Staphylococcus spp. most frequently involved in cases of subclinical mastitis is CoNS; ${ }^{7}$ infection with CoNS results in a significant increase in somatic cell counts and considerably decreased milk production. ${ }^{4}$ To identify the most efficient control strategy for this disease, it is highly important to perform agent identification and antibiograms to indicate which specific treatment should be used. ${ }^{8}$ Data regarding goat mastitis in Mexico are scarce; therefore, the results of this study represent important contributions to the body of knowledge necessary for understanding this disease. The objectives of this study were to identify mastitis-causing bacteria using traditional bacteriological methods in samples collected on goat farms in central Mexico (Guanajuato, Querétaro, Morelos and Mexico City) by milking type; and to obtain information regarding the health status of the mammary glands of these goats. Additionally, we also aimed to determine the susceptibility of the isolates to chemotherapeutic agents. 


\section{Materials and methods}

A total of 460 samples of goat milk were obtained via manual and mechanical milking from dairy farms with housing and semi-intensive systems in three different states in central Mexico (Querétaro, Morelos, and Guanajuato) and Mexico City. Milk samples were individually obtained from the glands of selected goats; the following goat breeds were included in this study: Alpine French, Saanen and Toggenburg. During the sampling process, the nipples were first disinfected using a $70 \%$ alcohol swab and then cleaned beginning at the nipple apex and continuing toward the gland (if the gland was very dirty, it was washed with $\mathrm{pH}$ neutral soap to remove the dirt). The first three milk squirts were manually extracted, and approximately $30 \mathrm{ml}$ samples were collected in new sterile and hermetic vials from each gland. Samples were kept under refrigeration and then frozen at $-10{ }^{\circ} \mathrm{C}$ and transferred to the microbiology laboratory. Samples were gradually defrosted by placed them in a refrigerator at $4{ }^{\circ} \mathrm{C}$ for 24 hours and then placing them in a water bath at $37^{\circ} \mathrm{C}$ for 15 minutes to complete defrosting. Once thawed, the samples were agitated using the electric stirrer until homogenization was achieved so that any bacteria that were trapped in fat globules were released. Once homogenized, the milk was seeded on trypticase soybean agar and MacConkey agar plates divided into four quadrants and incubated at $37{ }^{\circ} \mathrm{C}$ for 24 hours to obtain primary cultures. When no growth was observed, the plates were incubated for 48 more hours, after which the samples were considered negative. In samples that demonstrated bacterial growth, the morphologies of the colonies were macroscopically examined. Samples from the colonies were Gram-stained to observe the morphology, clustering and tinctorial affinity of the bacterial cells upon microscopic examination. If Gram-positive cocci were identified, catalase tests were performed to differentiate between the Staphylococcus spp. and Streptococcus spp. Colonies identified as Staphylococcus spp. were tested for coagulase via the tube test to determine whether they were positive coagulase ( $S$. aureus or $S$. intermedius) or CoNS. Each isolate was identified to the genus level and, in some cases, to the species level. The colonies were seeded again on trypticase soy agar using pure culture techniques for species identification. Staphylococcus species identification was performed using the API Staph ${ }^{\circledR}$ system according to the manufacturer's instructions. The plates were incubated for 24 hours at $37^{\circ} \mathrm{C}$, and numerical keys were obtained from the system and entered into the manufacturer's website to obtain identification information. The Staphylococcus colonies identified at the species level were tested for susceptibility to chemotherapeutic agents using the Kirby-Bauer technique. Each inoculum was standardized and adjusted to match an equivalent turbidity of 0.5 on the McFarland nephelometer scale and seeded into grooves on scored Müller-Hinton agar plates; afterward, sensi-discs were placed on each plate. The antibiotics evaluated in this study were as follows: polymyxin B, ampicillin, tobramycin, tetracycline and gentamicin; although only ampicillin is used as a treatment against mastitis, the rest of the antibiotics were donated by the Department of Medicine and Zootechnics of Ruminants of the FMVZ UNAM and were useful to know the susceptibility of the isolates to different types of antibiotics. The plates were incubated at $37^{\circ} \mathrm{C}$ for 24 hours; then, the susceptibility test results were interpreted. The information obtained in this study was evaluated via contingency table analyses and logistic regression models using the JMP 12.0 statistical package (SAS Institute Inc, 2015). Additionally, a cluster analysis was performed using Ward's minimum variance method and the DELL STATISTICA program (Data Analysis Software System).

\section{Results}

Among the three states and one city in which samples were obtained, Querétaro had 30 samples that were positive for bacterial growth, 11 positive samples were obtained from Guanajuato, 12 positive samples were obtained from Morelos and three positive samples were obtained from Mexico City. A total of 370 samples were obtained from farms that used mechanical milking, and 90 samples were obtained from farms that used manual milking. We observed that subclinical mastitis, as indicated using the aforementioned test, was significantly more prevalent in mechanically milked herds ( $\mathrm{P}$ $<0.01)$. Table 1 shows the states in which the samples were obtained, samples positive for bacterial growth and the percentage of samples that belonged to each one of these categories. Of the 370 samples obtained from farms employing mechanical milking, 47 were positive for bacterial growth, representing $12.70 \%$ of the total samples, whereas of the 90 samples obtained from farms employing manual milking, nine were positive for bacterial growth, representing $10 \%$ of the total samples. Of these 56 positive cases, 33 were identified as Staphylococcus, representing $58.92 \%$ of the bacteria-positive samples; 22 were Gram-negative rods (39.2\%), and one was identified as Micrococcus spp. (1.78\%). Of the 33 Staphylococcuspositive samples, 16 were identified as Staphylococcus epidermidis, representing $48.48 \%$ of the Staphylococcus-positive samples; 5 each were identified as Staphylococcus simulans and Staphylococcus xylosus, each representing $15.15 \%$ of the Staphylococcus-positive samples; 3 were identified as Staphylococcus lentus, representing $9.09 \%$ of the Staphylococcus-positive samples; 2 were identified as Staphylococcus aureus, representing $6.06 \%$ of the Staphylococcuspositive samples; and 1 each was identified as Staphylococcus caprae and Staphylococcus cohnii, each representing 3.03\% of the Staphylococcus-positive samples. Thirty-one of the 33 staphylococci were coagulase negative (93.9\%), representing $55.3 \%$ of the bacteriapositive cases overall. The 33 isolates identified as Staphylococcus spp. were tested for susceptibility to chemotherapeutic agents using polymyxin B, ampicillin, tobramycin, tetracycline and gentamycin; of these isolates, $13(39.39 \%)$ were susceptible to all antibiotics; $13(39.39 \%)$ had intermediate susceptibility to polymyxin B, 4 $(12.12 \%)$ exhibited resistance to polymyxin B; one (3.03\%) presented intermediate susceptibility to tobramycin; one (3.03\%) demonstrated intermediate susceptibility to polymyxin B and resistance to ampicillin; and one $(3.03 \%)$ showed resistance to polymyxin B and ampicillin. The isolates that presented intermediate susceptibility and resistance were $S$. epidermidis.

Table 1 Sampling by state and bacterial positivity

\begin{tabular}{llll}
\hline State & $\begin{array}{l}\text { Obtained } \\
\text { samples }\end{array}$ & $\begin{array}{l}\text { Positive samples } \\
\text { for bacterial } \\
\text { growth }\end{array}$ & $\begin{array}{l}\text { Percentage that } \\
\text { represents }\end{array}$ \\
\hline Querétaro & 262 & 30 & 6.52 \\
Guanajuato & 112 & 11 & 2.39 \\
Morelos & 50 & 12 & 2.6 \\
Mexico & 36 & 3 & 0.65 \\
City & 460 & 56 & 12.17 \\
Total & & & \\
\hline
\end{tabular}

\section{Discussion}

Several studies have reported Staphylococcus aureus to be the main pathogen that is most frequently isolated from bovine milk; 
however, several authors have shown that this is not the case for goat milk. Among these authors, ${ }^{6}$ reported CoNS to be the most frequently identified microorganism. In this study, CoNS were identified most frequently, coinciding with the results of previous studies conducted in Spain, Italy, Kenya, Argentina, Brazil, Venezuela ${ }^{9-14}$ and Mexico. ${ }^{15-}$ 17 These bacteria predominate in the mammary gland of goats, accounting for $55.3 \%$ of the 56 bacteria-positive samples identified in this study; these results are similar to the findings identified by Zavaleta et al., ${ }^{17}$ in the states of Coahuila and San Luis Potosí in northern Mexico, in which a detection rate of $60.91 \%$ was obtained, and with the study conducted by ${ }^{15}$ in Querétaro, wherein a detection rate of $63.9 \%$ was obtained. Similarly, Staphylococcus epidermidis was the pathogen that was most frequently identified in this study, with a detection rate of $48.48 \%$; this is dissimilar from the results identified in Mexican studies conducted by, ${ }^{8,17}$ wherein the most frequently observed Staphylococcus species were S. chromogenes and $S$. epidermidis, which were isolated from $1.6 \%$ and $1.14 \%$ of cases, respectively. Regarding manual and mechanical milking, contrary to all expectations, a slight increase in the prevalence of bacterial growth was identified in samples obtained from farms using mechanical milking $(12.7 \%)$ vs those using manual milking $(10 \%)$. These results are similar to those reported in a previous study, ${ }^{14}$ wherein no significant differences were identified between these two types of milking. The efficacy of beta lactam antibiotics has continued to be demonstrated in central Mexican goats, which have not have been found to have high levels of resistance to this type of antibiotics. In this work, we detected a $6.06 \%$ rate of resistance to these antibiotics; this is contrary to what has been identified in some other countries, such as Brazil, where 100\% resistance rates have been identified, ${ }^{13}$ or Italy with a $36 \%$ of resistance. ${ }^{10}$ However, intermediate susceptibility $(39.39 \%)$ and resistance $(18.18 \%)$ to polymyxin B were observed in the majority of samples in this study, and $S$. epidermidis was the bacterial agent that most frequently demonstrated intermediate susceptibility and resistance. These results are similar to those identified in another study wherein this pathogen was found to be resistant to six antimicrobials. ${ }^{10,18-20}$ Attention should be paid to the handling and maintenance of the milking equipment, as mastitis may occur due to overmilking, poor pulsation and pressure calibrations, and inadequate washing of milking equipment. In both mechanical and manual milking, hygiene is the main prevention strategy for bacterial mastitis. CoNS were most frequently identified as responsible for caprine subclinical mastitis in this study, which was conducted in the states of Morelos, Querétaro, and Guanajuato and Mexico City (58.92\%). The Staphylococcus species that was most frequently isolated was $S$. epidermidis $(48.48 \%$ ), followed by $S$. simulans and S. xylosus (15.15\%). S. aureus was isolated in 2 cases, representing only $6.06 \%$ of the Staphylococcus-positive samples. Among the CoNS, S. epidermidis is considered pathogenic in humans. In this study, this Staphylococcus species was the most frequently detected pathogen; therefore, it is very important to implement relevant preventive measures to decrease in its presence. Although we did not identify a high level of resistance to beta lactam antibiotics in this study, opposing results were identified for polymyxin B. The differences observed in the efficacies of the evaluated drugs illustrate the importance of carrying out tests for the identification of and evaluation of antimicrobial susceptibility in bacterial agents related to mastitis.

\section{Conclusions}

We these results, we conclude that subclinical mastitis is the most prevalent form of the disease in goats, especially in mechanical milking herds, and the bacterial genera most frequently isolated in goat milk were coagulase-negative Staphylococcus, mainly S. epidermidis. Although the CoNS are considering minor pathogens, it is necessary to investigate the virulence factors they possess to cause mastitis in dairy cattle. On the other hand, it is important to carry out studies to detect resistance genes to antibiotics, as the species identified show resistance to the antibiotics used in the antibiograms of this study.

\section{Acknowledgements}

None.

\section{Conflicts of interest}

Author declares there is no conflict of interest.

\section{References}

1. SIAP. Servicio de Información Agroalimentaria y Pesquera. Producción Nacional de leche; 2014.

2. FAO. Food and Agriculture Organization of the United Nations. Producción mundial de leche; 2016.

3. Koop G, Nielen M, Werven T. Diagnostic tools to monitor udder health in dairy goats. Vet Quart. 2012;32(1):37-44.

4. Bergonier D, Cremoux R, Rupp, et al. Mastitis of dairy small ruminants. Vet Res. 2003;34(5):689-716.

5. Hernández L. Enfermedades de las cabras. SAGARPA-INIFAP: México; 2015. p. 233-242

6. Contreras A, Sierra D, Sanchez A, et al. Mastitis in small ruminants Small Rumin Res. 2007;68(1-2):145-153.

7. Contreras A, Luengo C, Sánchez A, et al. The role of intramammary pathogens in dairy goats. Livest Prod Sci. 2003;79(2-3):273-283.

8. Ruiz R. Development of a molecular multiplex PCR tool for the detection of the main agents of caprine mastitis. National Autonomous University of Mexico: Mexico; 2010.

9. Luengo R, Sánchez L, Corrales R, et al. Lactational study of the factors of variation of the somatic cell count in milk of goats. SEOC. 2002. p. $700-706$.

10. Virdis S, Scarano C, Cossu F, et al. Antibiotic resistance in Staphylococcus aureus and coagulase negative staphylococci isolated from goats with subclinical mastitis. Vet Med Int. 2010;517060.

11. Ndegwa EN. Risk factors associated with subclinical subacut mastitis in Kenyan dairy goats. Isr J Vet Med. 2001;56:4-8.

12. Sticotti Sticotti E, Giraudo J, Mació, et al. Bacterial agents present in milk of goats with clinical mastitis in extensive breeding systems. Primer Congreso Caprino: Argentina; 2013.

13. Rodrigues da Silva E, Pimenta A, Dias J, et al. Identification and in vitro antimicrobial susceptibility of Staphylococcus species isolated from goat mastitis in the Northeast of Brazil. Small Ruminant Res. 2004;55(13):45-49.

14. Zambrano W, Sánchez J. Comparative study of mecanical and manual milking sistems in the mastitis clinical and subclinical development in Aroa. GCV 4: Yaracuy State; 1998. p. 62-74.

15. Ruiz R, Cervantes R, Ducoing A, et al. Principales géneros bacterianos aislados de leche de cabra en dos granjas del municipio de Tequisquiapan, Querétaro, México. Rev Mex Cienc Pecu. 2013;4(1):93-106.

16. Flores A. Identification of Staphylococcus aureus in milk of goats with clinical mastitis in the lagoon of Coahuila. Autonomous University of 
Aguascalientes: Mexico; 2012.

17. Zavaleta M, Hernández L, Díaz A, et al. Frecuencia de Staphylococcus coagulasa negativo en leche de cabra. XL Congreso Nacional de Buiatría; México; 2013.

18. Bedolla C. Mastitis Bovina. Cuatro Vientos: México; 2004.
19. Statistica, Dell statistica. Data Analysis Software System. USA; 2015.

20. Sánchez A, Contreras A, Corrales J, et al. Influencia de la infección intramamaria subclínica en la producción láctea de rebaños de cabras murciano granadinas. Med Vet. 1997;14:290-293. 